TRANSACTIONS OF THE

AMERICAN MATHEMATICAL SOCIETY

Volume 355, Number 10, Pages 3843-3859

S 0002-9947(03)03383-X

Article electronically published on June 24, 2003

\title{
MEROMORPHIC GROUPS
}

\author{
ANAND PILLAY AND THOMAS SCANLON
}

\begin{abstract}
We show that a connected group interpretable in a compact complex manifold (a meromorphic group) is definably an extension of a complex torus by a linear algebraic group, generalizing results of Fujiki. A special case of this result, as well as one of the ingredients in the proof, is that a strongly minimal modular meromorphic group is a complex torus, answering a question of Hrushovski. As a consequence, we show that a simple compact complex manifold has algebraic and Kummer dimension zero if and only if its generic type is trivial.
\end{abstract}

\section{IntroduCtion}

Let $\mathcal{A}$ be the category of reduced irreducible compact complex spaces. By a Zariski open subset of some $X \in \mathcal{A}$ we mean, as usual, the complement of an analytic subset of $X$. We understand a meromorphic mapping from $X$ to $Y(X, Y \in$ $\mathcal{A})$ in the sense of Remmert. Roughly speaking, it is an analytic subset $Z$ of $X \times Y$ such that for some (nonempty, so dense) Zariski open subset $U$ of $X, Z \cap(U \times Y)$ is the graph of a holomorphic function from $U$ to $Y$. $\mathcal{A}$ considered as a manysorted structure, whose sorts are the compact complex spaces and whose basic relations are the analytic subsets of various Cartesian products of the sorts, is a structure with quantifier-elimination and finite Morley rank (sort by sort). This was proved by Zilber [20] (although quantifier-elimination was also noted earlier in [12]). Quantifier-elimination says that the definable sets are precisely the finite unions of locally Zariski closed subsets of various compact complex spaces. It follows that definable functions are precisely piecewise meromorphic functions. Moreover, there is a natural complex analytic definition of the class of groups interpretable in a compact complex manifold.

Fujiki, in his study [4] of automorphism groups of compact Kähler manifolds, introduces the notion of a "meromorphic group". As we will be proposing a less restrictive meaning for "meromorphic group", we will refer to Fujiki's notion as "Fujiki-meromorphic". A Fujiki-meromorphic group is a complex Lie group G which is a Zariski open subset of some compact complex space $G^{*}$ such that the group operation of $G$ extends to a meromorphic mapping from $G^{*} \times G^{*}$ to $G^{*}$ which is holomorphic on $\left(G \times G^{*}\right) \cup\left(G^{*} \times G\right)$. Let $\mathcal{C}$ be the full subcategory of $\mathcal{A}$ consisting of compact complex spaces which are holomorphic images of compact Kähler manifolds. Fujiki proves that if $G$ is a connected Fujiki-meromorphic group in $\mathcal{C}$ (namely $G^{*} \in \mathcal{C}$ ), then $G$ is "meromorphically" isomorphic to an extension of

Received by the editors June 16, 2000.

2000 Mathematics Subject Classification. Primary 30Dxx.

The first author was partially supported by an NSF grant; the second, by an NSF MSPRF.

(C)2003 American Mathematical Society 
a complex torus by a linear algebraic group, generalizing Chevalley's well-known theorem for algebraic groups. He raises the issue of whether this remains true in the more general category $\mathcal{A}$, and proves it for $G$ commutative.

While we will show that every group interpretable in a compact complex manifold is definably isomorphic to a Fujiki-meromorphic group, such groups are naturally only meromorphic groups in the following less restrictive sense. A meromorphic group $G$ is a complex Lie group with a finite covering by Zariski open subsets $U_{i}$ of irreducible compact complex spaces $X_{i}(i=1, \ldots, n)$ such that both the transition maps and the group operation on $G$ extend to meromorphic maps between the various $X_{i}$ and their products. Note that if the $X_{i}$ happen to be algebraic varieties, then this agrees with the definition of an abstract algebraic group. Complex algebraic groups, complex tori, and Fujiki-meromorphic groups are all meromorphic groups. Using basic results on the model theory of compact complex manifolds, it is a routine matter to see that the class of meromorphic groups coincides with the class of groups interpretable in some compact complex manifold. Our results imply that meromorphic groups coincide with Fujiki-meromorphic groups, and moreover have Kähler compactifications.

A strongly minimal set in $\mathcal{A}$ is a definable set without infinite, co-infinite definable subsets. A strongly minimal group in $\mathcal{A}$ is precisely a meromorphic group without proper infinite Zariski closed subsets. In [10] it was noted that the deep results of [11] apply to strongly minimal sets definable in $\mathcal{A}$, implying that any strongly minimal definable group $G$ either is a (one-dimensional) algebraic group, or is modular: every definable subset of $G \times \cdots \times G$ is a finite Boolean combination of translates of subgroups. Simple complex tori of dimension $>1$ are examples of strongly minimal modular groups (see [14]). For the converse, Hrushovski [8] asked whether strongly minimal modular groups are (necessarily simple) complex tori. In fact, Hrushovski outlined to the first author some ideas for proving this, depending however on finding a good compactification of the group. In any case, the question was answered by the second author in [18] for the special case when $G$ is itself interpretable in a strongly minimal compact complex manifold. In 14 additional observations about $\mathcal{A}$ and its model theory were made, including elimination of imaginaries. Also, it was asked whether the Chevalley theorem holds for groups definable in $\mathcal{A}$. We found subsequently that the same question was asked in [4] for Fujiki-meromorphic groups.

We will prove the following results:

Theorem 1.1. Suppose $G$ is a strongly minimal meromorphic group. Then $G$ is meromorphically isomorphic to either a one-dimensional algebraic group or a simple nonalgebraic torus.

Theorem 1.2. Suppose $G$ is a connected meromorphic group. Then $G$ has a normal connected meromorphic subgroup $L$ such that $L$ is (meromorphically isomorphic to) a linear algebraic group, and $G / L$ is a complex torus. Moreover, $L$ is unique.

Theorem 1.1 is a special case of Theorem 1.2. Theorem 1.1 will be proved by finding a good compactification of $G$ (i.e. showing that $G$ is Fujiki-meromorphic) and then (as $G$ is commutative) referring to [4]. By again finding a suitable compactification we will first prove Theorem 1.2 for the special case when $G$ is an extension of a one-dimensional linear algebraic group by a simple complex torus. 
The general case will follow by an induction on dimension, making use of some additional ingredients such as the structure of compact complex spaces with algebraic codimension one, and some model theory of groups of finite Morley rank.

In the next section we give some definitions and recall both complex analytic and model-theoretic notions. In section 3 we carry out compactifications, proving Theorem 1.1 among other things. In section 4 we prove Theorem 1.2. Some additional remarks are made in section 5 .

\section{Preliminaries}

For basic results, notions and notation concerning complex spaces and meromorphic maps, we refer the reader to [3], [6] and [19]. However, we will repeat a few crucial definitions and results which we will be relying on. For us $\mathcal{A}$ denotes the class of reduced irreducible compact complex spaces. We take as given the notion of a holomorphic map $f$ from $X$ to $Y$, where $X, Y \in \mathcal{A}$. $\operatorname{dim}(X)$ denotes the complex dimension of $X$. A modification of $X \in \mathcal{A}$ is some $Y \in \mathcal{A}$ and a surjective holomorphic $f: Y \rightarrow X$ such that for some proper closed analytic subsets $A$ of $Y$ and $B$ of $X, f \mid(Y \backslash A): Y \backslash A \rightarrow X \backslash B$ is biholomorphic. Resolution of singularities says that any $X$ has a modification $(Y, f)$ such that $Y$ is nonsingular (so a connected compact complex manifold). The notion of a meromorphic mapping $f$ from $X$ to $Y(X, Y \in \mathcal{A})$ is crucial. Such an object can be defined in various equivalent ways. For $X$ irreducible we define $f$ to be a function from $X$ to the set of subsets of $Y$ such that the "graph" of $f,\{(x, y) \in X \times Y: y \in f(x)\}$ is an irreducible analytic subset of $X \times Y$, and for all $x$ in some (dense) Zariski open subset $U$ of $X, f(x)$ is a singleton. For a general $X$ we say that $f$ is meromorphic if each of its restrictions to the irreducible components of $X$ is meromorphic. We say that $f$ is holomorphic, or defined, at the points in $U$. Let $Z$ be the graph of $f$ as defined above, and $\pi$ the projection from $Z$ onto $X$. Then $(Z, \pi)$ turns out to be a modification of $X$. The projection of $Z$ on the second coordinate is then a holomorphic map from $Z$ to $Y$ which is said to be a resolution of indeterminacies of the meromorphic map $f$. From the definition of a meromorphic map one easily derives the following fact.

Fact 2.1. Let $X, Y \in \mathcal{A}$. Let $f, g$ be meromorphic mappings from $X$ to $Y$. Suppose that for some dense Zariski open subset $U$ of $X, f$ and $g$ agree on $U$. Then $f=g$.

Suppose that $U$ is a dense Zariski open subset of $X \in \mathcal{A}$, and $f$ a holomorphic map from $U$ into $Y \in \mathcal{A}$. By abuse of language we may sometimes say that $f$ is meromorphic if there is a meromorphic mapping $g$ from $X$ to $Y$ which agrees with $f$ on $U$. A natural category which can be associated to $\mathcal{A}$ is the category whose objects are those complex spaces which are Zariski open subsets of spaces in $\mathcal{A}$ and whose morphisms are the holomorphic maps which are meromorphic in the sense of the previous sentence. If we restrict our attention to those $X, Y$ which are projective algebraic varieties, this category is exactly that of quasiprojective varieties and morphisms.

It is also natural to consider complex spaces which have a finite covering by Zariski open subsets $U_{i}$ of spaces $X_{i}$ in $\mathcal{A}$ where the transition maps are meromorphic in the above sense. Morphisms in this category would be holomorphic maps which are meromorphic (in the above sense) when read in each $U_{i}$. From the model-theoretic point of view this is the category of complex spaces definable in $\mathcal{A}$ with holomorphic definable functions as the morphisms. What we will call a 
meromorphic group is exactly a group object in this latter category. Here is the precise definition.

Definition 2.2. A meromorphic group is a complex Lie group $G$, with a finite covering by open subsets $W_{i}$, for $i=1, \ldots, n$, and for each $i$ a (biholomorphic) isomorphism $\phi_{i}$ of $W_{i}$ with a Zariski open subset $U_{i}$ of some $X_{i} \in \mathcal{A}$ such that

(i) for each $i \neq j, \phi_{i}\left(W_{i} \cap W_{j}\right)$ is a Zariski open subset of $X_{i}$, and the induced biholomorphic map between $\phi_{i}\left(W_{i} \cap W_{j}\right)$ and $\phi_{j}\left(W_{i} \cap W_{j}\right)$ is meromorphic, namely is the restriction of a meromorphic mapping between $X_{i}$ and $X_{j}$; and

(ii) For each $i, j, k$, the set $\left\{(x, y) \in U_{i} \times U_{j}: \phi_{i}^{-1}(x) \cdot \phi_{j}^{-1}(y) \in W_{k}\right\}$ is Zariski open in $X_{i} \times X_{j}$ and the induced holomorphic map $((x, y)$ goes to $\left.\phi_{k}\left(\phi_{i}^{1}(x) \cdot \phi_{j}^{-1}(y)\right)\right)$ from $U_{i} \times U_{j}$ to $U_{k}$ is meromorphic, namely is the restriction of a meromorphic mapping between $X_{i} \times X_{j}$ and $X_{k}$.

Conditions (i) and (ii) can be expressed briefly by saying that the transition maps as well as the group operation are meromorphic when read in the various $U_{i}$ and their Cartesian products.

We say that the covering by the $W_{i}$ 's and the isomorphisms with the $U_{i}$ 's satisfying (i) and (ii) above give the complex Lie group $G$ a meromorphic structure.

If $G$ are a meromorphic group as in Definition 2.2, by a meromorphic subgroup $H$ of $G$ we mean a closed subgroup such that for each $i, \phi_{i}\left(H \cap W_{i}\right)$ is the intersection of an analytic subset of $X_{i}$ with $U_{i}$. Clearly $H$ has the structure of a meromorphic group.

A holomorphic homomorphism (complex Lie homomorphism) $f$ between meromorphic groups $G_{1}$ and $G_{2}$ is meromorphic if when restricted to the charts the map is meromorphic, that is, extends to meromorphic mappings between the relevant compact complex spaces.

So now we have the category of meromorphic groups and meromorphic homomorphisms. The following says that quotient objects exist. It follows from looking at the equivalent category of definable groups and using the elimination of imaginaries result from [14. This will be explained below.

Fact 2.3. Let $G$ be a meromorphic group and $N$ a normal meromorphic subgroup. Then there are a meromorphic group $H$ and a surjective meromorphic homomorphism from $G$ to $H$ whose kernel is $N$.

We now repeat the definition of a Fujiki-meromorphic group and recall what Fujiki proved.

Definition 2.4. Let $G$ be a complex Lie group.

(i) A meromorphic compactification of $G$ is a compact complex space $G^{*} \in \mathcal{A}$ which contains $G$ as a dense Zariski open subset, such that the group operation $\mu: G \times G \rightarrow G$ is meromorphic, i.e. the restriction of a meromorphic mapping $\mu^{*}$, say, from $G^{*} \times G^{*}$ to $G^{*}$

(ii) A Fujiki compactification of $G$ is a meromorphic compactification $\left(G^{*}, \mu^{*}\right)$ of $G$ such that $\mu^{*}$ is holomorphic on $\left(G \times G^{*}\right) \cup\left(G^{*} \cup G\right)$.

(iii) $G$ is Fujiki-meromorphic if $G$ has a Fujiki compactification.

Remark 2.5. (i) A Fujiki-meromorphic group is a meromorphic group.

(ii) A connected compact complex Lie group (i.e. a complex torus) is Fujikimeromorphic. 
(iii) (Remark 2.3 of [4.) A complex algebraic group is Fujiki-meromorphic.

Following the notation of Fujiki [4, we set up

Definition 2.6. We will call the meromorphic group $G$ regular if there is a meromorphic homomorphism $f$ from $G^{0}$, the connected component of the identity in $G$, onto a complex torus $T$ such that the kernel $L$ of $f$ is meromorphically isomorphic to a connected linear algebraic group. (Briefly said: $G^{0}$ is meromorphically an extension of a complex torus $T$ by a linear algebraic group $L$.)

Remark 2.7. (i) Let $G$ be regular and let $T, L$ be as above. Then $L$ and $T$ are unique. In particular, $L$ is the unique maximal normal connected meromorphic subgroup of $G^{0}$ which is meromorphically isomorphic to a linear algebraic group.

(ii) A regular meromorphic group is Fujiki-meromorphic.

Proof. (i) Suppose $L_{1}$ is a normal connected meromorphic subgroup of $G$ which is meromorphically isomorphic to a linear algebraic group. Then $L_{1} / L$ meromorphically embeds in $T$. So $L_{1} / L$ is both a complex torus and a linear algebraic group, forcing it to be trivial. That is, $L_{1}$ is contained in $L$.

(ii) As in Remark 2.3 of [4].

Recall that $\mathcal{C}$ is the subclass (in fact, the full subcategory) of $\mathcal{A}$ consisting of those $X$ which are holomorphic images of compact connected Kähler manifolds. We will say that the connected meromorphic group $G$ is of type $\mathcal{C}$ if there is a Fujiki compactification $G^{*}$ of $G$ which is in $\mathcal{C}$. Fujiki proves:

Fact 2.8. (i) A Fujiki-meromorphic group $G$ is regular iff $G$ is of type $\mathcal{C}$.

(ii) If $G$ is commutative and Fujiki-meromorphic, then $G$ is regular.

In the final part of this section we discuss the model theory of compact complex manifolds. We will have to assume the basics of model theory, and a bit more. [7] is a good reference for basic model theory. The first four chapters of [2] (by Bouscaren, Ziegler, Lascar, Pillay) are a useful reference for various aspects of applied and geometric stability theory. [15] is an advanced text on geometric stability. [1] deals with the theory of groups of finite Morley rank. Another good reference for stable groups is [17].

We consider $\mathcal{A}$ as a many-sorted first order structure whose sorts are the (reduced, irreducible) compact complex spaces and whose basic relations are the analytic subsets of finite Cartesian products of such things.

Fact 2.9. $\operatorname{Th}(\mathcal{A})$ has quantifier-elimination and elimination of imaginaries, and each sort has finite Morley rank. Moreover, $\mathcal{A}$ is $\aleph_{1}$-compact.

Quantifier-elimination was proved in [12], and independently in [20]. It says that any definable subset of a sort $X$ is analytically constructible, that is, a finite union of intersections of analytic (Zariski closed) subsets and complements of analytic (Zariski open) sets. A characterization of definable functions follows from this: Suppose $U$ is a definable set, and $f$ a definable function from $X$ into some sort $Y$. Then we can write $X$ as a disjoint union of definable sets $U_{i}$, where each $U_{i}$ is a Zariski open subset of some sort (complex space) $X_{i}$ such that for each $i$, the restriction of $f$ to $U_{i}$ is holomorphic and is the restriction to $U_{i}$ of a meromorphic mapping from $X_{i}$ to $Y$. We say, with possibly some abuse of language, that definable functions are piecewise meromorphic. 
Zilber [20] proved finiteness of Morley rank. Elimination of imaginaries was observed in [14].

$\aleph_{1}$-compactness means that any countable family of definable subsets of some sort $X$ has nonempty intersection as long a every finite subfamily does.

With Fact 2.9 there is a remarkable parallel between complex-analytic and model-theoretic structural and classification results. We refer the reader to [20], 8], 14 for more discussion. Note that on the face of it $\mathcal{A}$ is not $\aleph_{1}$-saturated, as each element of each sort is essentially named by a constant. One can ask whether there is some sublanguage $L_{0}$ of the full language $L$ described above such that every relation in $L$ is definable, possibly with parameters, in the language $L_{0}$ and such that the reduct $\mathcal{A} \mid L_{0}$ is $\aleph_{1}$-saturated. This is not true, as, for example, a general generalized Hopf surface has continuum many holomorphic automorphisms, but our Proposition 5.2 shows that it has trivial generic type and hence cannot have an infinite definable family of automorphisms. On the other hand, $\mathcal{C}$ can be considered as a reduct of $\mathcal{A}$ (fewer sorts but the full structure on each sort), which has quantifier-elimination and elimination of imaginaries in its own right. Fujiki's results [5] on the Douady spaces of manifolds in $\mathcal{C}$ imply that the structure $\mathcal{C}$ is $\aleph_{1}$-saturated in a suitable sublanguage. (See [13].)

$\mathcal{A}^{\prime}$ will denote a very saturated elementary extension of $\mathcal{A}$. For any $X \in \mathcal{A}, X^{\prime}$ denotes its canonical extension in $\mathcal{A}^{\prime}$. We will often work model-theoretically in $\mathcal{A}^{\prime}$. For example, a definable property holds generically on $X$ iff it holds for a generic point of $X^{\prime}$.

A definable group $G$ in $\mathcal{A}$ will be called connected if $G$ has no definable subgroups of finite index. Any meromorphic group is clearly a definable group (using elimination of imaginaries). Methods from the algebraic case due to Hrushovski and van den Dries (see [17] as well as Pillay's article in [2]) adapt to yield the important

Fact 2.10. Any interpretable group $G$ in $\mathcal{A}$ is definably isomorphic to a meromorphic group $H$ (unique up to meromorphic isomorphism).

This fact gives a natural equivalence between the category of definable groups and that of meromorphic groups. In particular any definable homomorphism between meromorphic groups will be meromorphic (and, hence, by homogeneity, holomorphic). From here on we will use "definable" interchangeably with "meromorphic" when talking about groups and homomorphisms.

A definable set $X$ (in $\mathcal{A}$ ) is said to be strongly minimal if $X$ is infinite and has no infinite co-infinite definable subsets. A definable connected group $A$ is said to be modular if every definable subset of $A^{n}$ is a Boolean combination of translates of definable subgroups. In [10] it was proved that the results of [11] apply to the category $\mathcal{A}$. This yields

Fact 2.11. Suppose $G$ is a definable connected group in $\mathcal{A}$ which has no infinite normal definable subgroups. Then either $G$ is strongly minimal and modular, or $G$ is definably isomorphic to a (complex) algebraic group.

It follows that if $T$ is a nonalgebraic simple complex torus, then $T$ is modular. (A direct proof, avoiding [11], was given in [14].) 


\section{Compactifications}

We will prove

Theorem 3.1. Let $G$ be a connected commutative meromorphic group which is either strongly minimal, or an extension of a connected one-dimensional linear algebraic group by a simple complex torus. Then $G$ is Fujiki-meromorphic.

A consequence is:

Corollary 3.2. (i) Let $G$ be a strongly minimal meromorphic group. Then $G$ is meromorphically isomorphic to either a one-dimensional algebraic group or a simple modular complex torus.

(ii) Let $G$ be a commutative meromorphic group which is an extension of a onedimensional linear algebraic group by a simple complex torus. Then $G$ meromorphically splits.

Proof. (i) $G$ is commutative, so by Theorem 3.1, Fujiki meromorphic, and thus, by Fact 2.8 (ii), meromorphically an extension of a complex torus $T$ by a linear algebraic group $L$. As $G$ is strongly minimal, $G$ is either $T$ or $L$. If $G=L$, then $\operatorname{dim}(L)=1$. If $G=T$, then $T$ has no proper infinite analytic subsets, so $T$ is either an elliptic curve or simple and modular (by 2.11).

(ii) Immediate, by Fact 2.8 (ii).

To prove Theorem 3.1, we will find a meromorphic compactification $G^{*}$ of $G$ and then show it to be a Fujiki compactification. The following general result concerning compactifications of commutative meromorphic groups will be useful.

Lemma 3.3. Suppose that the connected commutative meromorphic group $(G, \mu)$ has meromorphic compactification $\left(G^{*}, \mu^{*}\right)$. Suppose $S=G^{*} \backslash G$ is nonempty. Then:

(i) Every component of $S$ has codimension 1 in $G^{*}$.

(ii) $\mu^{*} \mid\left(G^{*} \times S\right)$ is a meromorphic mapping from $G^{*} \times S$ to $S$.

(iii) For each $g \in G$ and each component $C$ of $S, \mu_{g}^{*}=\mu^{*}(g,-): C \rightarrow C$ is biholomorphic on a dense Zariski open subset of $C$, and for $g, h \in G$ we have $\mu_{g}^{*} \cdot \mu_{h}^{*}=\mu_{g . h}^{*}$ on a dense Zariski open subset of $C$.

Proof. (i) Let $n=\operatorname{dim}\left(G^{*}\right)(=\operatorname{dim}(G))$. Suppose for the sake of contradiction that there is $x \in S$ such that $\operatorname{dim}_{x}(S)<n-1$. Let $\Delta_{n}$ be the open unit disc in $\mathbb{C}^{n}$ and let $f: \Delta_{n} \rightarrow U$ be a coordinate function for any open neighborhood $U$ of $x$ in $G^{*}$, where $U$ is chosen such that $U \cap S$ has dimension $<n-1$. So if $A=f^{-1}(U \cap S)$, then $A$ is an analytic subset of $\Delta_{n}$ of codimension at least 2 , and $f_{1}=f \mid\left(\Delta_{n} \backslash A\right)$ is a holomorphic embedding into $G$. As $G$ is a connected commutative Lie group, its universal cover is $\pi: \mathbb{C}^{n} \rightarrow G$. As $A$ has codimension at least two in $\Delta_{n}, \Delta_{n} \backslash A$ is simply connected, and so $f_{1}$ lifts to a holomorphic map $f_{2}: \Delta_{n} \rightarrow \mathbb{C}^{n}$ (see [3], Section 2.23). Let $g=\pi \circ f_{2}$. Then $g$ is a holomorphic map from $\Delta$ into $G^{*}$ which agrees with $f$ off the thin analytic subset $A$. But then, as $f$ and $g$ are holomorphic functions which agree generically, we have $f=g$, contradicting the fact that $x \notin G$. (i) is proved.

(ii) Let $\Gamma$ be the graph of $\mu^{*}$. We will first show that for all $(g, x)$ in some dense Zariski open subset $V$ of $G^{*} \times S,\{y:(g, x, y) \in \Gamma\}$ is finite. If not, then for a Zariski open subset $V$ of $G^{*} \times S$, the above set of $y$ 's has positive dimension. It 
follows from (i) that $\operatorname{dim}\left(\Gamma \cap\left(G^{*} \times S \times G^{*}\right)\right) \geq 2 n$, contradicting irreducibility of $\Gamma$. It now follows by the implicit function theorem that

(a) $\mu^{*}$ is holomorphic on $V$.

For $g \in G$ let $\mu_{g}^{*}$ be $\mu^{*}(g,-)$, a meromorphic mapping from $G^{*}$ to $G^{*}$. Note that if $\mu_{g}^{*}$ is defined (single valued) at $x$ and $\mu_{h}^{*}$ is defined at $\mu_{g}^{*}(x)$, then $\mu_{h g}^{*}$ is defined at $x$ and equals $\mu_{h}^{*}\left(\mu_{g}^{*}(x)\right)$. It follows that if $(g, x) \in V$ and $g \in G$, then $\mu(g, x) \in S$. As $G$ is Zariski dense in $G^{*}$ it follows that

(b) $\mu^{*} \mid\left(G^{*} \times S\right)$ is a meromorphic mapping into $S$, yielding (ii).

(iii) The same argument as above shows that for any $g \in G, \mu_{g}^{*} \mid S$ is a meromorphic mapping from $S$ to $S$. Let $C_{1}, \ldots, C_{s}$ be the irreducible components of $S$. Note that the image of the meromorphic mapping $\mu_{g}^{*}$ from $G^{*}$ to $G^{*}$ (i.e. the projection of its graph on the second component) is all of $G^{*}$. But for $x \in G, \mu_{g}^{*}(x) \in G$. Thus the image of the meromorphic mapping $\mu_{g}^{*} \mid S$ is all of $S$. We work modeltheoretically. Fix $C_{i}$. Let $x$ be a generic point of $C_{i}^{\prime}$ over $\mathcal{A}$. So $y=\mu_{g}^{*}(x) \in C_{j}^{\prime}$ for some $j=f(i)$. It follows that $\mu_{g}^{*} \mid C_{i}$ is a meromorphic mapping from $C_{i}$ into $C_{f(i)}$.

(c) Thus $f=f_{g}$ must be a permutation of $\{1, \ldots, s\}$.

If for some $i$, and $x$ as above, $\mu_{g}^{*}(x)$ is not a generic point of $C_{f(i)}^{\prime}$ over $\mathcal{A}$, then there is a proper analytic subset $D_{f(i)}$ of $C_{f(i)}$ such that $\mu_{g}^{*} \mid C_{i}$ has image contained in $D_{f(i)}$. By (c), we contradict the fact that $\mu_{g}^{*} \mid S$ has image all of $S$.

Thus for $x \in C_{i}^{\prime}$ generic, $\mu_{g}^{*}(x)$ is generic in $C_{f(i)}^{\prime}$ over $\mathcal{A}$. It follows that $g \rightarrow f_{g}$ gives a definable action of $G$ on $\{1, \ldots, s\}$. As $G$ is connected, this has to be trivial. This gives (iii).

Remark 3.4. (iii) above is interpreted model-theoretically by saying that $G$ acts generically on $C$ : let $p=p_{C}$ be the generic type of the component $C$ of $S$. Then for $g \in G^{\prime}$ and $x$ realizing $p$ independent of $g$ (over $\mathcal{A}$ ), $\mu^{*}(g, x)$ is defined, realizes $p$ and is independent from $g$. Moreover, if $g, h \in G^{\prime}$ and $x$ realizes $p$ independent of $g, h$, then $\mu^{*}(h g, x)=\mu^{*}\left(h, \mu^{*}(g, x)\right)$.

We can now obtain the strongly minimal case of Theorem 3.1.

Lemma 3.5. Let $G$ be a strongly minimal meromorphic group. Then $G$ is Fujikimeromorphic.

Proof. Step 1. Finding a meromorphic compactification.

By assumption, on $G$ some open nonempty definable subset $U$ of $G$ is already a Zariski open subset of a compact complex space $X$, which we may assume by resolution of singularities to be a manifold. Moreover, by strong minimality of $G$, $G \backslash U$ is finite, say $\left\{g_{1}, \ldots, g_{n}\right\}$. For $i=1, \ldots, n$ let $V_{i}$ be a coordinate neighborhood of $g_{i}$ in $G$ such that the closures $\bar{V}_{i}$ of the $V_{i}$ in $G$ are disjoint. Note that $K_{i}=$ $\bar{V}_{i} \backslash\left\{g_{i}\right\}$ is contained in $U$, so in $X$, but is not compact, so not closed in $X$. Let $D_{i}$ be the boundary of $K_{i}$ in $X$, namely $\bar{K}_{i} \backslash K_{i}$. Let $\pi: X \rightarrow X^{\prime}$ be the quotient map which collapses each $D_{i}$ to a point $c_{i}$. Then $X^{\prime}$ is compact, $\pi$ is holomorphic (in fact is a modification), and is biholomorphic outside the union of the $D_{i}$ 's. Let $f: G \rightarrow X^{\prime}$ be defined by $f(x)=\pi(x)$ for $x \in U$ and $f\left(g_{i}\right)=c_{i}$. Then $f$ is a definable, holomorphic embedding.

Step 2. $G^{*}$ is a Fujiki compactification of $G$.

If $G=G^{*}$, there is nothing to do. Otherwise, (as $G$ is commutative) Lemma 3.3 applies. Let $S$ be as there. We will show that $G$ is holomorphic on $S$, and in fact acts as the identity. Note that the generic type of $G$ is orthogonal to any 
set of dimension less than that of $G$ ( $G$ being strongly minimal). In particular, $G$ is orthogonal to $S$. Fix a component $C$ of $S$. Lemma 3.3 (iii) gives us a generic action of $G$ on $C$. Let $g, h \in G^{\prime}$ be generic independent elements of $G$ and let $x$ be generic in $C^{\prime}$ over $\{g, h\}$. Then by the orthogonality mentioned above, each of $g$ and $h$ is independent from $\left\{x, \mu^{*}(g, x)\right\}$. It follows that $\mu^{*}(g, x)=\mu^{*}(h, x)$, and thus $\mu^{*}\left(h^{-1} \cdot g, x\right)=x$. But $h^{-1} . g$ is generic in $G^{\prime}$ and independent from $x$. It follows that $G$ acts generically trivially on $C$. So the holomorphic map from $G^{*} \times C$ to $C$ taking $(g, x)$ to $x$ agrees generically with the meromorphic mapping $\mu^{*} \mid\left(G^{*} \times C\right): G^{*} \times C \rightarrow C$. By Fact 2.1, these mappings agree. This shows that $\left(G^{*}, \mu^{*}\right)$ is a Fujiki compactification of $G$.

We now deal with the case when $G$ is a commutative extension of the additive group $\mathbb{G}_{a}$ or the multiplicative group $\mathbb{G}_{m}$ by a simple complex torus $T$. We let $H$ denote $G / T$ (so $H$ is $\mathbb{G}_{a}$ or $\mathbb{G}_{m}$ ). If $G$ is meromorphically isomorphic to an algebraic group, then $G$ is clearly Fujiki-meromorphic (in fact the Chevalley theorem applies immediately, yielding Theorem 1.2). If $T$ has a definable complement in $G$ (up to finite), then again we get the required conclusion. So for the rest of this section we make:

Assumption.

(a) $G$ is a commutative meromorphic group which is meromorphically an extension of $H$ by a simple complex torus $T$, where $H$ is $\mathbb{G}_{a}$ or $\mathbb{G}_{m}$.

(b) $G$ is not meromorphically isomorphic to an algebraic group.

(c) There is no definable connected infinite subgroup $L$ of $G$ with $L \cap T$ finite.

We will show that $G$ is Fujiki-meromorphic (which actually leads to a contradiction).

We will make use of the socle theory from [9].

Lemma 3.6. $T$ is the maximal almost pluriminimal definable subgroup of $G$.

Proof. Note that $T$, being simple, is almost strongly minimal. So if the lemma fails, as $G / T$ has dimension 1, $G$ is semipluriminimal. By [9, $G$ is an almost direct product of pairwise orthogonal semiminimal groups. If $G$ is already semiminimal, then as $G$ is nonorthogonal to $\mathbb{P}^{1}$ via $G \rightarrow H, G$ must be algebraic, contradicting Assumption (b) Thus $G$ is the semidirect product of $T$ and some $L$, contradicting Assumption (c).

Lemma 3.7. Let $X$ be a definable subset of $G$. Assume that the Morley rank of $X$ is strictly less than the Morley rank of $G$ (equivalently, $X$ is not Zariski dense in $G)$. Then $X$ is contained in finitely many translates of $T$.

Proof. We prove the lemma by induction on $R M(X)=m$. It is clearly true for $m=0$. We may assume that the Morley degree of $X$ is 1 . Let $S$ be the (modeltheoretic) stabilizer of $X$. If $S$ is finite, then by Lemma 3.6, as well as Proposition 4.3 of [9], $X$ is, up to a set of Morley rank $<m$, contained in a single translate of $T$. By the induction hypothesis, $X$ is contained in finitely many translates of $T$, as desired. So we may assume that $S$ is infinite. By Assumption (c), and the fact that $T$ is simple, $S$ must contain $T$. Note that $R M(T)=R M(G)-1 \geq R M(X)$. But it is well-known that the Morley rank of the stabilizer of a Morley degree 1 set $X$ is at most the Morley rank of $X$, with equality if and only if the stabilizer is connected and $X$ is, up to a set of smaller Morley rank, a translate of this stabilizer. 
Thus $R M(S)=R M(X), S=T$, and, up to a set of smaller Morley rank, $X$ is a translate of $T$, so we finish again by induction.

Lemma 3.8. $G$ is Fujiki-meromorphic.

Proof. As in the strongly minimal case we first find a compact complex manifold $G^{*}$ containing $G$ as a Zariski open set, and then show that this gives $G$ a Fujikimeromorphic structure.

Step I. Finding the compactification.

Let $R M(G)=n$. By definition of $G$ being a meromorphic group, let $U$ be a definable subset (with Morley rank $n$ ) of $G$ which is a dense Zariski-open subset of a compact complex manifold $\bar{U}$. Let $\pi: G \rightarrow H$ be the canonical surjective homomorphism. Then $\pi$ takes $U$ onto a cofinite subset $\pi(U)$ of $H$.

Claim 1. We may assume that for any $x \in \pi(U), \pi^{-1}(x) \cap U=\pi^{-1}(x)$ (a translate of $T)$.

Proof. $Y=\pi^{-1}(\pi(U)) \backslash U$ is a definable subset of $G$ of Morley rank $<n=R M(G)$. By Lemma 3.7, $Y$ is contained in finitely many translates of $T$, namely finitely many fibers of $\pi$. Remove these fibers from $U$.

Let $\pi^{\prime}$ denote $\pi \mid U . \quad \pi^{\prime}$ extends to a meromorphic function $\bar{\pi}$ from $\bar{U}$ to $\mathbb{P}^{1}$. Further restricting $U$, we may assume:

Claim 2. For all $x \in \pi^{\prime}(U),\left(\pi^{\prime}\right)^{-1}(x)=\bar{\pi}^{-1}(x)$.

Let $C$ be the finite set $H \backslash \pi(U)$. Then we can find $h \in \pi(U)$ such that $h . C \subset$ $\pi(U)$. Let $g \in U$ be a preimage of $h$. Let $\tau_{g}: G \rightarrow G$ be multiplication by $g . \tau_{g} \mid U$ is not defined everywhere, but is holomorphic on the open set where it is defined, and so extends to a meromorphic map $\bar{\tau}_{g}: \bar{U} \rightarrow \bar{U}$. By a theorem of Remmert (see Theorem 1.9 in Chapter VII of [6]), there are a modification $\nu: \tilde{U} \rightarrow \bar{U}$ and a holomorphic map $\tilde{\tau}: \tilde{U} \rightarrow \bar{U}$ such that $\tilde{\tau}=\bar{\tau}_{g} \cdot \nu$. In particular, for $x \in \tilde{U}$ such that $\tau_{g} \mid U$ is defined at $\nu(x)$, we have $\tilde{\tau}(x)=\tau_{g}(\nu(x))$.

Claim 3. $\bar{\pi} \cdot \tilde{\tau}=\tau_{h} \cdot \bar{\pi} \cdot \nu$.

Proof. This holds generically, so holds everywhere.

We will now construct the required compactification $G^{*}$ of $G$ as a holomorphic image of $\tilde{U}$. Let $S=\mathbb{P}^{1} \backslash H$. So $S=\{\infty\}$ or $\{\infty, 0\}$. As a set, $G^{*}$ will be the disjoint union of $G$ with $\bar{\pi}^{-1}(S)$. The manifold structure of $G^{*}$ is as follows: $G$ is given its canonical manifold structure. Now let $x \in \bar{\pi}^{-1}(S)$. Let $y=\bar{\pi}(x) \in \mathbb{P}^{1}$. Choose an open neighborhood $V$ of $y$ in $\mathbb{P}^{1}$ such that $V \backslash\{y\} \subset U$. Then $\bar{\pi}^{-1}(V) \subset G^{*}$ is an open neighborhood of $x$. The transition maps are clearly holomorphic, yielding a structure of a complex compact manifold on $G^{*}$ containing $G$ as an open (dense) subset.

Now we define a holomorphic surjective map $f$ from $\tilde{U}$ to $G^{*}$. Let $x \in \tilde{U}$. If $\bar{\pi}(\tilde{\tau}(x)) \notin C$, define $f(x)=\tilde{\tau}(x)$ (so $f(x) \in \bar{\pi}^{-1}(\pi(U) \cup S) \subset G^{*}$ ). On the other hand, if $\bar{\pi}(\tilde{\tau}(x)) \in C$, define $f(x)=g . \nu(x)$. Note that in this latter case we have $\nu(x) \in U \subset G$, and so $g . \nu(x) \in G$ and $\pi(g . \nu(x))=\bar{\pi} \tilde{\tau}(x)$.

It is easy to check, given our assumptions, that $f$ is holomorphic and surjective. So $G^{*}$ is a compact complex manifold, containing $G$ as a dense Zariski open subset (the embedding of $G$ in $G^{*}$ is definable and holomorphic).

This completes Step I. 
Step II: The regular action of $G$ on itself extends to a trivial action on the boundary $G^{*} \backslash G$.

Let $C_{1}, \ldots, C_{k}$ be the irreducible components of $G^{*} \backslash G$. Note that for each $i$, $\operatorname{dim}\left(C_{i}\right)=\operatorname{dim}(T)$.

Claim 4. For each $i$ there is a surjective holomorphic map from $C_{i}$ to $T$ (so finiteto-one outside a proper Zariski closed subset $D_{i}$ of $C_{i}$ ).

Proof. By Step I we have a surjective holomorphic map $\pi: G^{*} \rightarrow \mathbb{P}^{1}$ such that $\pi^{-1}(H)=G$ and $\pi \mid G$ is precisely the canonical surjective homomorphism from $G$ to $H$. So $G^{*} \backslash G$ is $\pi^{-1}(S)$, where $S=\mathbb{P}^{1} \backslash H$. Consider the map $\mu(g, h)=g \cdot h^{-1}$ from $G \times{ }_{H} G$ to $T$. This is definable and holomorphic, so it extends to a meromorphic map from $G^{*} \times_{\mathbb{P}^{1}} G^{*}$ to $T$, which we also call $\mu$. By Lemma 3.3 of [4] this map is holomorphic. In particular, for any $C_{i}$ and $x \in C_{i}, \mu(-, x) \mid C_{i}$ is a holomorphic map from $C_{i}$ into $T$. We must show that for suitable $x \in C_{i}$, this is surjective.

For $g \in G$, let $f_{g}$ be the meromorphic map from $G^{*}$ to $G^{*}$ whose restriction to $G$ is multiplication by $g$ (so $f_{g}$ is $\bar{\tau}_{g}$ in our previous notation).

Let $t \in T$. The map taking $x \in G$ to $\mu(t \cdot x, x) \in T$ is the constant map with value $t$. It follows that whenever $x \in G^{*}$ and $f_{t}$ is single valued at $x$, then $\mu\left(f_{t}(x), x\right)=t$. Choose $x_{0}$ generic in $C_{i}$. Then for a dense open set $V$ of $t$ 's in $T, f_{t}\left(x_{0}\right)$ is defined and in $C_{i}$. So for each $t \in V, \mu\left(f_{t}\left(x_{0}\right), x_{0}\right)=t$. Thus $\mu\left(-, x_{0}\right) \mid C_{i}: C_{i} \rightarrow T$ is generically surjective, so surjective. In any case this map is finite.

By Lemma 3.3, for each $i, f_{g}$ induces a generic holomorphic action of $G$ on $C_{i}$. Let $K_{i}$ be the subgroup of $G$ consisting of those $g \in G$ such that for all $x$ in some Zariski open subset of $C_{i}, f_{g}(x)=x$. For dimension reasons $K_{i}$ is a definable infinite subgroup of $G$, so contains $T$. Moreover we have an induced generic action of $G / K_{i}$ on $C_{i}$. Let $D_{i}$ be as in Claim 4 . Let $x_{0} \in C_{i}$ be such that for any $h$ in some dense Zariski open subset of $G / K_{i}, h . x_{0} \in C_{i} \backslash D_{i}$. This gives a meromorphic map from $G / K_{i}$ to $C_{i}$ whose image contains infinitely many points outside $D_{i}$. Composing with the holomorphic map from $C_{i}$ to $T$ given by Claim 4 yields a meromorphic nonconstant map from $\mathbb{P}^{1}$ into $T$, which is impossible.

Thus $K_{i}=G$, and the generic action of $G$ on $C_{i}$ is trivial. This holds for each $i$. So the generic action of $G$ on $G^{*} \backslash G$ is trivial. That is, if $g \in G$, then the meromorphic mapping $f_{g}: G^{*} \rightarrow\left(G^{*} \backslash G\right)$ agrees with the identity map on a dense Zariski open set. This implies that $f_{g}$ is the identity map. So $G^{*}$ witnesses $G$ being Fujiki meromorphic. The proof of Lemma 3.8 is complete, as well as the proofs of Theorem 3.1 and Corollary 3.2

\section{Composition Series}

In this section we will prove

Theorem 4.1. Suppose $G$ is a connected meromorphic group. Then $G$ is regular (in the sense of Definition 2.6).

We first state a consequence of Theorem 3.1:

Proposition 4.2. Suppose the connected meromorphic group $G$ is simple, in the sense that $G$ has no nontrivial, connected normal definable subgroup. Then $G$ is either

(i) an (almost simple) noncommutative algebraic group, 
(ii) $\mathbb{G}_{a}$ or $\mathbb{G}_{m}$,

(iii) a simple abelian variety, or

(iv) a strongly minimal modular complex torus.

Proof. Simplicity of $G$, together with the the dichotomy theorem from [1], implies that either $G$ is nonorthogonal to $\mathbb{P}^{1}$ (namely has nontrivial algebraic reduction) or $G$ is modular. In the first case, $G$ is an algebraic group, so (i), (ii) or (iii) holds. In the second case, every definable subset of $G$ is a Boolean combination of cosets of definable subgroups. Simplicity implies that $G$ is strongly minimal. By $3.2, G$ is a complex torus.

The following will be crucial. The special case for Fujiki-meromorphic groups in the class $\mathcal{C}$ was proved in [4]. In any case the classical theory of groups of finite Morley rank enters the picture.

Lemma 4.3. Let $1 \rightarrow L \rightarrow G \rightarrow H \rightarrow 1$ be an exact sequence of connected meromorphic groups and suppose that $L$ and $H$ are (meromorphically isomorphic to) linear algebraic groups. Then so is $G$.

Proof. Note that if $G$ satisfies the hypotheses of the lemma and $G_{1}$ is a connected definable subgroup of $G$, or an image of $G$ under a meromorphic homomorphism, then $G_{1}$ satisfies the hypotheses too (for suitable $L_{1}, H_{1}$ ).

We will prove the lemma by induction on $\operatorname{dim}(G)=n$. We consider various possibilities for $G$,

Case I. $G$ has an an infinite center.

By the hypotheses, $Z(G)$ contains an infinite definable linear algebraic group and thus, by the structure of commutative linear algebraic groups, $Z(G)$ contains a definable 1-dimensional connected linear algebraic group $A$. $A$ is normal in $G$, so by the induction hypothesis $G / A$ is (meromorphically isomorphic to) a linear algebraic group. It makes sense to talk about the algebraic dimension $a(G)$ of $G$. Note that $\operatorname{dim}(G / A)=n-1$, so the $\operatorname{map} G \rightarrow G / A$ witnesses that $a(G) \geq n-1$. If $a(G)=n$, then $G$ is already isomorphic to an algebraic group, so a linear algebraic group. Otherwise $a(G)=n-1$, and it is well-known (see [19]) that the general fiber of the algebraic reduction $\pi: G \rightarrow X$ is an elliptic curve $E$. But the map $G \rightarrow G / A$ must meromorphically factor through $\pi$. The general fiber of the first map is $\mathbb{P}^{1}$, and thus we see that $E$ is an image of $\mathbb{P}^{1}$ under a meromorphic (i.e. rational) map, a contradiction. Thus $G$ is linear algebraic.

Case II. $G$ is solvable.

We may assume, by Case I, that $Z(G)$ is finite. But then $G / Z(G)$ is centerless, and easily $G$ is linear algebraic iff $G / Z(G)$ is. So we may assume that $G$ is centerless. As is shown in Chapter 3 of [17] or Chapter 9 of [1], the commutator subgroup $G^{\prime}$ of $G$ is connected, and nilpotent, so $Z\left(G^{\prime}\right)$ is infinite and contains a minimal definable connected $G$-normal subgroup $A$. $G / G^{\prime}$ defines an infinite group of automorphisms of $A$. Again by results in [17] or [1], $A$ is the additive group of a definable field $K$. As $A$ is by hypothesis linear algebraic, the field $K$ has to be (definably isomorphic to) $\mathbb{C}$ and $\operatorname{dim}(A)=1 . G / A$ is by the induction hypothesis algebraic, and as in Case 1 we deduce that $G$ is (definably) algebraic.

Case III. $G$ is nonsolvable.

Note that $G$ is among other things a connected complex Lie group, and as such we have the Levi-Malcev decomposition $G=R . S$, where $R$ is the maximal normal 
solvable connected subgroup of $G, S$ is a semisimple (complex) Lie group (unique up to conjugacy in $G$ ), and $R \cap S$ is discrete. Finite Morley rank considerations (see 5.38 in [1]) show that $R$ is definable, so linear algebraic by Case II (or induction). It is probably then well-known that $G$ must be isomorphic as a complex Lie group to a linear algebraic group. However, we want $G$ to be definably isomorphic to such a group. So we must do a little more work, although maybe there is a more direct way. We will show

Claim. $S$ is a definable subgroup of $G$.

Proof. We may assume that $R$ is a proper, nontrivial subgroup of $G$, definably isomorphic to a linear algebraic group. We will first reduce to the case where $R$ is commutative and unipotent. Let $H$ be the connected component of the center of the commutator subgroup of $R$. $H$ is then a nontrivial commutative connected linear algebraic group, normal in $G$. So $H$ is the direct product $U . T$ of a commutative unipotent group $U$ and an algebraic torus $T$. Note that both $U$ and $T$ are definable connected normal subgroups of $G$. $T$ has no infinite definable group of automorphisms, so is central in $G$. By Case I we may assume $T$ to be trivial. Thus $H=U$ is unipotent. By the induction hypothesis, $G / H$ is linear algebraic. Clearly $R / H$ is the solvable radical of $G / H$. Thus $G / H$ is an almost direct product of $R / H$ with a semisimple algebraic group $G_{1} / H$ (where $G_{1}$ is a definable connected subgroup of $G$ containing $H$ ). As $S$ is unique up to conjugacy, we may assume that $G_{1}=H . S$. Note that the homomorphism $\mu: G_{1} \rightarrow G_{1} / H$ is an isomorphism on $S$.

Note that $G_{1} / H$ is linear algebraic, by the induction hypothesis among other things. Now $S$ (being semisimple) is isomorphic (uniquely) as a complex Lie group to a linear algebraic group, so it makes sense to talk about an element of $S$ being unipotent. $S$ is an almost direct product of almost simple groups $S_{1}, \ldots, S_{r}$. Fix a nontrivial unipotent element $a$ in some $S_{i} \backslash H$. Work now inside the definable group $G_{1}$. Let $a_{1}=\mu(a) \in G_{1} / H$. $a_{1}$ is then unipotent, and let $U_{1}$ be a 1-dimensional definable unipotent subgroup of $G_{1} / H$ containing $a_{1}$. Let $U_{2}=\mu^{-1}\left(U_{1}\right)$. Then $U_{2}$ is an extension of a unipotent linear algebraic group $\left(U_{1}\right)$ by a linear algebraic unipotent group $H$, so it is (by induction) linear algebraic unipotent. $a \in U_{2}$. We can find a definable commutative connected subgroup $U_{3}$ of $U_{2}$ containing $a$. $U_{3}$ is definably a vector space over $\mathbf{C}$. The 1 -dimensional subspace $U_{4}$ generated by $a$ is a definable subgroup of $G$ contained in $S_{i}$. We have found an infinite connected subgroup $U_{4}$ of $S_{i}$ which is definable in $G$. The group generated by all the $U_{4}^{g}$, where $g \in S_{i}$, is definable and must be equal to $S_{i}$. So $S_{i}$ is definable. As $i$ was arbitrary, $S$ is definable. The claim is proved.

We want $S$ to be definably isomorphic to a linear algebraic group. Recall that $S$ as a complex Lie group is the almost direct product of almost simple (discrete center) groups $S_{1}, \ldots, S_{r}$. As the center of $S$ is definable, each $S_{i}$ has finite center. By considering centralizers, each $S_{i}$ is definable. Also, $S_{i}$, being almost simple, is almost strongly minimal, hence, by the validity of the Zilber trichotomy in $\mathcal{A}$, modular or nonorthogonal to $\mathbb{P}^{1}$. But $S_{i}$ is nonabelian. So it must be nonorthogonal to $\mathbb{P}^{1}$, so algebraic. Thus $S$ is definably an algebraic group, so by semismplicity, linear algebraic.

Finally $G$, being the almost semidirect product of linear algebraic $R$ with linear algebraic $S$, must be linear algebraic. Case III is complete, as well as the proof of Lemma 4.3 . 
Proof of Theorem 4.1. The proof will by induction on $\operatorname{dim}(G)$.

We first deal with the case when $G$ is commutative. Let $H$ be a minimal definable connected subgroup of $G$. By $4.2, H$ is either (a) a linear algebraic group or (b) a simple complex torus. If $G=H$, we are finished. Otherwise, applying the induction hypotheses, $G / H$ is definably an extension of a complex torus $T$, by a linear algebraic group $L / H$. In case (a), by Lemma $4.3, L$ is linear algebraic. So $G$ is definably an extension of $T$ by $L$, and we finish. So suppose (b) holds. If $L / H$ is trivial, $G$ is an extension of a complex torus by a complex torus, so also a complex torus. Otherwise let $L_{1} / H$ be a 1-dimensional subgroup of $L / H$. Then $L$ is definably an extension of $\mathbb{G}_{a}$ or $\mathbb{G}_{m}$ by the simple complex torus $T$. By Corollary 3.2 (ii) $L$ splits, yielding a 1-dimensional linear algebraic subgroup of $G$. We are now back in case (a). This proves Case I.

We now deal with the general case.

If $G$ has no proper normal nontrivial definable connected subgroup, then we are finished by 4.2

Otherwise let $H$ be a proper normal nontrivial connected subgroup of $G$. The induction hypothesis applies to $H$. If the maximal connected linear algebraic normal subgroup $L$ of $H$ is nontrivial, then $L$ is normal in $G$, as $L$ is characteristic in $H$ by Remark 2.7(i), and by applying the induction hypothesis to $G / L$ and applying Lemma 4.3 we finish. Otherwise $H$ is a complex torus. As a complex torus has no infinite definable group of automorphisms, $H$ is central in $G$. The induction hypothesis applies to $G / H$. If the latter is a complex torus, so is $G$. Otherwise $G$ has a connected normal definable subgroup $G_{1}$ containing $H$ such that $G_{1} / H$ is linear algebraic. If $G_{1} / H$ is semisimple (equivalently, contains no infinite normal solvable subgroup), then $G_{1}$ is the almost direct product of its commutator subgroup $G_{1}^{\prime}$ and $H . G_{1}^{\prime}$ is semisimple, so (definably) linear algebraic. We conclude by applying the induction hypothesis to $G / G_{1}^{\prime}$ and using Lemma 4.3.

If $G_{1} / H$ is not semisimple, then there is a definable nontrivial connected subgroup $A$ of $G_{1}$ containing $H$ such that $A$ is normal in $G$ and $A / H$ is commutative (and linear algebraic). $A / H$ is (definably) a product of $\mathbb{G}_{a}$ 's and $\mathbb{G}_{m}$ 's. Remember that $H$ is central in $A$, so if $A$ is not commutative, then the commutator map yields a nonconstant meromorphic map from $A / H \times A / H$ into the complex torus $H$, which is impossible. So $A$ has to be commutative. By the induction hypothesis, or by the first part of the proof (if $A=G$ ), $A$ has a definable connected (nontrivial) subgroup which is normal in $G$ and (definably) linear algebraic. By the induction hypothesis and Lemma 4.3, we finish.

This completes the proof of Theorem 4.1.

\section{Additional Remarks AND QUeStions}

We call a compact complex manifold (or space) $X$ simple if there is no definable family $\left\{Y_{t}: t \in T\right\}$ of positive-dimensional proper analytic subvarieties of $X$ such that $\bigcup\left\{Y_{t}: t \in T\right\}$ contains a Zariski open subset of $X$. It is easy to see that $X$ is simple if and only if its generic type $p_{X}$ has $U$-rank 1 , that is, is a minimal type. Recall that a minimal type $p(x)$ is trivial if any set of pairwise independent realizations of $p$ is an independent set. A simple compact complex manifold $X$ has a trivial generic type if and only if every analytic subvariety of any Cartesian power $X^{n}$ of $X$ is an intersection of subvarieties of the form $\pi^{-1}(Y)$, where $Y \subseteq X^{2}$ is an 
analytic subvariety of the Cartesian square of $X$ and $\pi: X^{n} \rightarrow X^{2}$ is a projection of the form $\left(x_{1}, \ldots, x_{n}\right) \mapsto\left(x_{i}, x_{j}\right)$ for some $i \leq j$.

The following was first proved by the second author [18]. We give a quick proof using Theorem 1.1.

Proposition 5.1. Let $X$ be a strongly minimal compact complex manifold. Then $X$ is either a (smooth projective) algebraic curve, a complex torus, or has trivial generic type.

Proof. Suppose that $X$ is neither trivial, nor an algebraic curve. Then $\operatorname{dim}(X)>1$, and by [10] and [1], there is a strongly minimal group $G$ definable in $X$. By Theorem $1.1 G$ must be a simple complex torus, nonorthogonal to $X$. Nonorthogonality is witnessed by an analytic subset $\Gamma$ of $X \times A$ which projects generically finite-toone on each of $X$ and $G$. As both $X, G$ are strongly minimal, both projections $\pi_{1}: \Gamma \rightarrow X$ and $\pi_{2}: \Gamma \rightarrow G$ are finite-to-one, and $\Gamma$ is strongly minimal with $\operatorname{dim}(\Gamma)=\operatorname{dim}(X)=\operatorname{dim}(G)>1$. Replacing $\Gamma$ by its normalization, we may assume $\Gamma$ is normal. $\Gamma$ has no proper infinite analytic subsets, in particular no codimension 1 analytic subsets. By the purity-of-branch theorem, $\pi_{2}$ is an unramified covering. As a finite unramified covering of a simple complex torus is necessarily a simple complex torus (since a subgroup of finite index in a lattice is also a lattice), $\Gamma$ is a complex torus. Via an easy calculation involving automorphisms of complex tori, one sees that the only finite groups of automorphisms which act freely on simple complex tori are given by translations by finite subgroups. Thus, if $T$ is a simple complex torus and $T \rightarrow Z$ is a finite unramified covering, then $Z$ is also a complex torus. As $\pi_{1}$ is also an unramified covering, $X$ is a complex torus.

We can more generally give natural necessary and sufficient conditions for a type of $U$-rank 1 to be trivial. A Kummer manifold is a compact complex space which is bimeromorphic with a space of the form $T / G$, where $T$ is a complex torus and $G$ a finite group of (holomorphic) automorphisms of $T$. Recall that the Kummer dimension of a compact complex manifold $X$ is the maximal dimension of a Kummer manifold $Y$ for which there is a dominant meromorphic map from $X$ to $Y$ (if such exists), and zero otherwise.

The following proposition explains the trichotomy within simple compact complex spaces between algebraic curves, nonalgebraic Kummer manifolds, and manifolds of zero algebraic and Kummer dimension in terms of the Zilber trichotomy for Zariski geometries.

Proposition 5.2. Suppose $X$ is a simple compact complex manifold. Then $p_{X}$ is trivial if and only if

(i) the algebraic dimension $a(X)$ of $X$ is 0 , and

(ii) there is no surjective meromorphic map from $X$ to a nontrivial Kummer manifold (i.e., $k(X)=0$, in the notation of [5]).

In particular, if $X \notin \mathcal{C}$, then $p_{X}$ is trivial.

Proof. $(\Leftarrow)$ : Suppose $p_{X}$ is nonorthogonal to $\mathbb{P}^{1}$. Then clearly $a(X)>0$. Suppose $p_{X}$ is nontrivial and modular. Then $p_{X}$ is nonorthogonal to the generic type of a strongly minimal modular torus $T$. Let $a$ be a generic point of $X^{\prime}$ (i.e. a realization of $\left.p_{X}\right)$. Then there is a generic $b \in T^{\prime}$ in $\operatorname{acl}(a)$. Let $\left\{b_{1}, \ldots, b_{n}\right\}$ be the finite set of realizations of $\operatorname{tp}(b / a)$. Then by the modularity of $T,\left(b_{1}, \ldots, b_{n}\right)$ is a generic point of a translate of a (strongly minimal) subtorus $S$ of $T^{n}$. After translating we 
may assume that $\left(b_{1}, \ldots, b_{n}\right)$ is a generic point of $S$. By elimination of imaginaries the finite set $\left\{b_{1}, \ldots, b_{n}\right\}$ is coded by some $c$. As $c \in \operatorname{dcl}(a)$, we obtain a surjective meromorphic map $f$ from $X$ to a compact complex manifold $Y$, where $c$ is a generic point of $Y$. But the map taking $\left(b_{1}, \cdots, b_{n}\right)$ to $c$ extends to a meromorphic map from $S$ to $Y$. Modularity of $S$ implies that this induces a bimeromorphic map between $S / G$ and $Y$ for some finite group $G$ of automorphisms of $S$. Thus $Y$ is Kummer. So if (i) and (ii) hold, the only possibility left for $p_{X}$ is to be trivial.

$\left(\Rightarrow\right.$ ): If (i) fails, then $p_{X}$ is nonorthogonal to $\mathbb{P}^{1}$, so is nontrivial. If (ii) fails and there is a surjective meromorphic map to the Kummer manifold $Y$, then clearly $Y$ is also simple and its generic type is nontrivial. So $p_{X}$ is nontrivial.

The "in particular" clause follows from the observations that any Kummer manifold $Y$ is in $\mathcal{C}$ as well as any compact complex manifold which maps meromorphically and generically finite-to-one on $Y$.

Our classification of meromorphic groups together with Fact 2.8 (i) shows that any meromorphic group $G$ is of type $\mathcal{C}$, that is, already definable in the structure $\mathcal{C}$. As $\mathcal{C}$ is saturated in a suitable language, our results also classify definable groups in all models of $\operatorname{Th}(\mathcal{C})$. What about groups definable in elementary extensions of $\mathcal{A}$ ? Here is a conjecture:

Conjecture 5.3. Let $G$ be a definable group in $\mathcal{A}^{\prime}$. Then $G$ is definably (in $\mathcal{A}^{\prime}$ ) isomorphic to a group $H$ definable in the reduct $\mathcal{C}^{\prime}$.

The conjecture can be restated in terms of families of groups in $\mathcal{A}$.

\section{NOTE ADDED IN PROOF}

An example refuting Conjecture 5.3 is presented in $[16$.

\section{REFERENCES}

[1] A. Borovik and A. Nesin, Groups of Finite Morley Rank, Oxford Logic Guides, Oxford University Press, 1994. MR 96c:20004

[2] E. Bouscaren (ed.), Model Theory and Algebraic Geometry, Lecture Notes in Math. 1696, Springer 1998. MR 99k:03032

[3] Gerd Fischer, Complex Analytic Geometry, Lecture Notes in Math. 538, Springer, 1976. MR 55:3291

[4] A. Fujiki, On automorphism groups of compact Kähler manifolds, Inv. Math., 44 (1978), 225-258. MR 58:1285

[5] A. Fujiki, Structure of manifolds in $\mathcal{C}$, in Algebraic Varieties and Analytic varieties (ed. H. Morikawa), Advanced Studies in Pure Mathematics 1, North-Holland, 1983, pp. 231-302. MR 85g:32045b

[6] H. Grauert, Th. Peternell, R. Remmert (eds.), Several Complex Variables VII, Springer 1994. MR 96k:32001

[7] W. Hodges, Model Theory, Cambridge University Press, 1993. MR 94e:03002

[8] E. Hrushovski, Geometric Model Theory, Proceedings of ICM 1998, vol. 1, Documenta Mathematicae 1998, extra vol. 1, 281-302. MR 2000b:03120

[9] E. Hrushovski, The Mordell-Lang conjecture for function fields, Journal AMS, 9 (1996), 667690. MR 97h:11154

[10] E. Hrushovski and B. Zilber, Zariski geometries, Bulletin AMS, 28(1993), 315-322. MR 93j:14003

[11] E. Hrushovski and B. Zilber, Zariski geometries, Journal AMS, 9 (1996), 1-56. MR 96c:03077

[12] S. Lojasiewicz, Introduction to complex analytic geometry, Birkhäuser, 1991. MR 92g:32002

[13] R. Moosa, Contributions to the model theory of fields and compact complex spaces, Ph.D. thesis. University of Illinois at Urbana-Champaign, 2001. 
[14] A. Pillay, Some model theory of compact complex spaces, Hilbert's tenth problem: relations with arithmetic and algebraic geometry (Ghent, 1999), 323-338, Contemp. Math., 270, AMS, Providence, RI, 2000. MR 2001m:03076

[15] A. Pillay, Geometric Stability Theory, Oxford University Press, 1996. MR 98a:03049

[16] A. Pillay and T. Scanlon, Compact complex manifolds with the DOP and other properties, J. Symbolic Logic, 67 (2002), no. 2, 737-743.

[17] B. Poizat, Groupes stables, Nur al-Mantiq wal-Marifah, Lyon, 1987. MR 89b:03056

[18] T. Scanlon, Locally modular groups in compact complex manifolds, preprint 2000.

[19] K. Ueno, Classification Theory of Algebraic Varieties and Compact Complex Spaces, Lecture Notes in Math., 439, Springer 1975. MR 58:22062

[20] B. Zilber, Model theory and algebraic geometry, Proceedings of 10th Easter conference (Wendisch Rietz, 1993; M. Weese and H. Wolter, eds.), Seminarbericht 93-1, Fachber. Math., Humboldt Univ., Berlin, 1993, pp. 202-222. MR 94i:03045

Department of Mathematics, University of Illinois at Urbana-Champaign, Urbana, ILLINOIS 61801

E-mail address: pillay@math.uiuc.edu

Department of Mathematics, University of California at Berkeley, Berkeley, CalIFORNIA 94720-3840

E-mail address: scanlon@math.berkeley.edu 